\title{
Adaptive Backstepping Control for a 2-DOF Robot Manipulator: A State Augmentation Approach
}

\author{
Nazila Nikdel and Mohammad Ali Badamchizadeh
}

\begin{abstract}
In this paper, the state augmented adaptive backstepping controller is designed and implemented on a two degrees of freedom (2-DOF) nonlinear robot manipulator. The controller should be designed in a way to stabilize the system and make the manipulator track the desired trajectory. The concept of backstepping control is to propose a virtual control signal in order to derive the tracking error value to zero. To this mean, first the system dynamic equations are simulated. The tracking error equations are derived and in order to improve the desired path tracking, an additional state is augmented to the equations. The state augmented backstepping controller is then developed for the system. The dynamical parameters of the manipulator are assumed to be unknown and an adaption law is derived via adaptive backstepping mechanism. Simulation results present the strength of the state augmented adaptive backstepping in tracking the desired trajectory.
\end{abstract}

Index Terms-2-DOF robot manipulator, adaptive backstepping, state augmentation.

\section{INTRODUCTION}

During the past years, different robotic systems are introduced and have made great developments [1], [2] and robotic manipulation has become an inseparable section of many areas such as military services, industries and medication [3], [4]. However nonlinear features usually restrict the manipulator's capability to do high-precision manipulation. One of the most challenging issues in robotic engineering is to face the system nonlinearity as well as parameter variations or uncertainties caused by inaccurate system modeling, insufficient parameter identification, external disturbances and environmental conditions. Control techniques should be developed in a way that make the robotic system adapt to these variations and be able to do the manipulation task [5]. To this mean variant control techniques have been applied on the manipulators. Different control methods such as conventional PID controllers, intelligent control methods, neural network based controllers and fuzzy control have been studied and implemented on the robotic systems [6]-[8]. Unfortunately, in existence of unknown parameter values or unstructured dynamical features, most of the traditional design approaches fail to succeed in obtaining the zero tracking error for the system [9]. Adaptive control approach can be used to deal with this issue. The adaptive structure of this type of controllers make them good candidates for facing dynamical parameter variations and

Manuscript received April 27, 2015; revised January 8, 2016.

The author are with the Faculty of Electrical and Computer Engineering, University of Tabriz, Tabriz 51666-14766, Iran (e-mail: n_nikdel@tabrizu.ac.ir, mbadamchi@ tabrizu.ac.ir). making the manipulator track the desired path despite the system nonlinear dynamics and parameter uncertainties. System stabilization is another important issue. The designed controller should satisfy the stability of the controlled system in existence of model nonlinearity and the system variable structure. Lyapunov theorem is a very strong tool proving the stability of the controlled manipulator by proposing a positive decreasing energy function [10]. The controller should adapt its structure to confront the parameter variations and despite its adaptive structure it should prove the stability of the controlled system. One of the most powerful nonlinear control strategies is the backstepping which proves the stability of the system via Lyapunov theorem. The backstepping and adaptive backstepping controllers have been used in a variety of research [11]-[13].

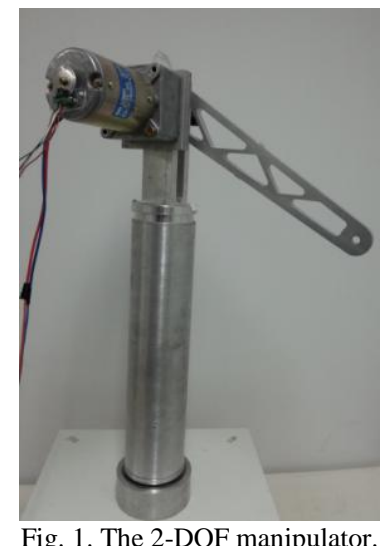

In this paper, a two-degree of freedom (2-DOF) manipulator with nonlinear dynamic equations is regarded as the case study and backstepping, state augmented backstepping and state augmented adaptive backstepping controllers are designed to control the vehicle. The controllers have to confront system nonlinearity while decreasing the tracking error value to zero. Despite dynamical parameters uncertainties, the stability of the system as well as good tracking performance should be guaranteed. In order to improve the tracking performance of the system an additional state is augmented to the system error equations [14] and the order of the equation system is increased. An adaption law is derived which stabilizes the system based on Lyapunov theorem. Finally after obtaining the Lyapunov function and the adaption law, the controller is implemented on the system. End-effector path tracking problem is investigated and the results are compared for the backstepping, the state augmented backstepping and the state augmented adaptive backstepping approaches.

The paper is organized as follows: Section II is devoted to the 2-DOF manipulator dynamic equations. Section III deals 
with the backstepping mechanism and discusses the uncertainty problem and an adaption law is derived via state augmented adaptive backstepping control approach. Simulation results are presented in Section IV. Section V concludes the paper.

\section{MANIPULATOR DYNAMIC EQUATIONS}

In this paper a 2-DOF manipulator, is regarded as the case study. The system is presented in Fig. 1. As the figure presents the system is a 2-link rotary manipulator. The frames are selected based on Denavit-Hartenberg (DH) convention [15] and forward and inverse kinematics are derived. By defining $q$ as the vector of joint variable. $M(q)$ is the inertia matrix of the entire manipulator. $C(q, \dot{q})$ is the matrix of Coriolis and centrifugal generalized forces. $G(q)$ is the gravity vectors and $\tau$ is the torque vector. The manipulator dynamic equation is as follows,

$$
M(q, \dot{q}) \ddot{q}+C(q, \dot{q}) \dot{q}+G(q)=\tau
$$

\section{APPLIED CONTROL APPROACHES}

\section{A. Backstepping Control}

Backstepping is a control approach which is applicable for a special type of nonlinear systems. The combination of the Lyapunov control theory and backstepping control guarantees the stability of the closed-loop system as well as its effective performance. In order to design the backstepping controller, the system equations are first decomposed into subsystems of less order. Backstepping has a recursive structure and in each step a Lyapunov function and a virtual control law are derived for each subsystem. As the final step, all the controller laws are integrated as the control law for the system and a Lyapunov function is introduced which satisfies the stability of the whole system. As the first step the error equation is derived based on (1). $e$ is defined as the tracking error and $q_{d}$ is the desired angle value. By taking the derivative of the error the equations will be,

$$
\begin{gathered}
e=q-q_{d} \\
z_{1}=e, \quad \dot{z}_{1}=z_{2}=\dot{e}=\dot{q}-\dot{q}_{d}, \quad \dot{z}_{2}=\ddot{e}=\ddot{q}-\ddot{q}_{d}
\end{gathered}
$$

The order of the system of the equations is two and is equal to the order of dynamic equations set (1). By expanding $\dot{z}_{2}$ based on (1),

$$
\begin{gathered}
\dot{z}_{2}=\ddot{q}-\ddot{q}_{d}=M^{-1}(\tau+\theta \varphi) \\
\theta \varphi=-M \ddot{q}_{d}-C \dot{q}-G
\end{gathered}
$$

In order to design the backstepping controller, the equation system (3) is separated into subsystems. The first equation subsystem is,

$$
\dot{z}_{1}=z_{2}
$$

$z_{2}$ is regarded as the virtual control signal for this subsystem.

Theorem I. Let $x_{e}=0$ be the equilibrium of the system $\dot{x}=f(x)$. If there exists a positive-definite function $V(x)>0$ which is continuously differentiable such that $V(0)=0$ and $\dot{V}(x)$ is negative-definite $(\dot{V}(x)<0)$, then $x_{e}$ is stable [10].

By defining the Lyapunov function for (6) as (7). By replacing the virtual control signal as $z_{2}=-k_{p} z_{1}$ where $k_{p}$ is a positive gain, the derivative of (7) will be (8),

$$
\begin{gathered}
V_{1}=\frac{1}{2} z_{1}^{2} \\
\dot{V}_{1}(z)=-k_{p} z_{1}^{2}<0
\end{gathered}
$$

So based on Theorem I. the virtual control can stabilize the subsystem (6). Finally for the whole system equations (3) the Lyapunov function is regarded as,

$$
V_{2}(z)=\frac{1}{2} z_{1}^{2}+\frac{1}{2} M\left(z_{2}+k_{p} z_{1}\right)^{2}
$$

Which is a positive-definite function. Taking the derivative of (9) will result in,

$$
\dot{V}_{2}(z)=z_{1} \dot{z}_{1}+M\left(z_{2}+k_{p} z_{1}\right)\left(\dot{z}_{2}+k_{p} \dot{z}_{1}\right)
$$

By expanding (10) based on (3) and (4) and defining the control signal $\tau$ as,

$$
\tau=-\theta \varphi-M k p z_{2}-z_{1}-k_{d}\left(z_{2}+k_{p} z_{1}\right)
$$

where $k_{d}$ is a positive gain. The derivative of Lyapunov function (10) will be,

$$
\dot{V}_{2}=-k_{p} z_{1}^{2}-k_{d}\left(z_{2}+k_{p} z_{1}\right)^{2}
$$

Which is a negative definite function. So, based on Theorem I., (9) and (12) the system (3) is stabilized by defining the control law as (11).

\section{B. State Augmented Backstepping Control}

The system dynamic (1) and the error equations (3) are of order 2. In order to enhance the controller performance, an additional state is augmented to the system equations. (3) is modified by adding the new state as the error integral and defining $x_{1}, x_{2}$ and $x_{3}$ as,

$$
x_{1}=\int_{0}^{t} e d t, x_{2}=e, x_{3}=\dot{e}
$$

By taking derivate of $x_{3}$ and according to (4) and (5),

$$
\dot{x}_{3}=\ddot{e}=M^{-1}(\tau+\theta \varphi)
$$

So the new state equations are, 


$$
\dot{x}_{1}=x_{2}, \quad \dot{x}_{2}=x_{3}, \quad \dot{x}_{3}=M^{-1}(\tau+\theta \varphi)
$$

First the augmented backstepping controller is designed and then it is expanded to the adaptive case. The equations should be decomposed into subsystems. The first subsystem is similar to (6) and the stability of the equation is satisfied based on the Lyapunov function $V=\frac{1}{2} k_{i} x_{1}^{2}$ where $k_{i}$ is a positive gain. By defining the virtual control signal as $x_{2}=-x_{1}$, the derivative of Lyapunov function will be $\dot{V}=-k_{i} x_{1}^{2}$ which is a negative function. Now consider the second subsystem as,

$$
\dot{x}_{1}=x_{2}, \dot{x}_{2}=x_{3}
$$

The Lyapunov function is defined as,

$$
V_{3}(x)=\frac{1}{2} k_{i} x_{1}^{2}+\frac{1}{2} x_{2}^{2}
$$

Taking the Lyapunov function derivative,

$$
\dot{V}_{3}(x)=k_{i} x_{1} \dot{x}_{1}+x_{2} \dot{x}_{2}=k_{i} x_{1} x_{2}+x_{2} x_{3}
$$

The virtual control signal $x_{3}$ is considered as $x_{3}=-k_{i} x_{1}-k_{p} x_{2}$, substituting $x_{3}$ in (18) will result in,

$$
\dot{V}_{3}(x)=-k_{p} x_{2}^{2}
$$

Which is a negative function and based on Theorem I. the second subsystem is stabilized utilizing the defined virtual control signal. As the final step the whole system equation set (15) is considered and the real control signal should be designed in a way to stabilize the system. The new Lyapunov function is defined as,

$$
\begin{aligned}
V_{4}(x)=\frac{1}{2} k_{i} & x_{1}^{2}+\frac{1}{2} x_{2}^{2} \\
& +\frac{1}{2} M\left(x_{3}+k_{p} x_{2}+k_{i} x_{1}\right)^{2}
\end{aligned}
$$

Since the inertia matrix $M(q)$ is always positive, the introduced Lyapunov function is also positive. Taking the derivative of the function will result in,

$$
\begin{aligned}
& \dot{V}_{4}(x)=k_{i} x_{1} \dot{x}_{1}+x_{2} \dot{x}_{2} \\
& \quad+M\left(x_{3}+k_{p} x_{2}+k_{i} x_{1}\right)\left(\dot{x}_{3}+k_{p} \dot{x}_{2}+k_{i} \dot{x}_{1}\right)
\end{aligned}
$$

The equation is simplified by substituting $\dot{x}_{1}, \dot{x}_{2}$ and $\dot{x}_{3}$ based on (15),

$$
\begin{aligned}
& \dot{V}_{4}(x)=k_{i} x_{1} x_{2}+x_{2} x_{3}+ \\
& \left(x_{3}+k_{p} x_{2}+k_{i} x_{1}\right)\left(\tau+\theta \varphi+M k_{p} x_{3}+M k_{i} x_{2}\right.
\end{aligned}
$$

The control signal $\tau$ is defined as,

$$
\begin{aligned}
\tau=-\theta \varphi-M k p x_{3} & -M k_{i} x_{2}-x_{2} \\
& -k_{d}\left(x_{3}+k_{p} x_{2}+k_{i} x_{1}\right)
\end{aligned}
$$

Equation (22) is rewritten by replacing $\tau$ with (23),

$$
\dot{V}_{4}(x)=-k_{p} x_{2}^{2}-k_{d}\left(x_{3}+k_{p} x_{2}+k_{i} x_{1}\right)^{2}
$$

Which is a negative function. So the designed control signal (23) is able to stabilize the system based on (20) and (24).

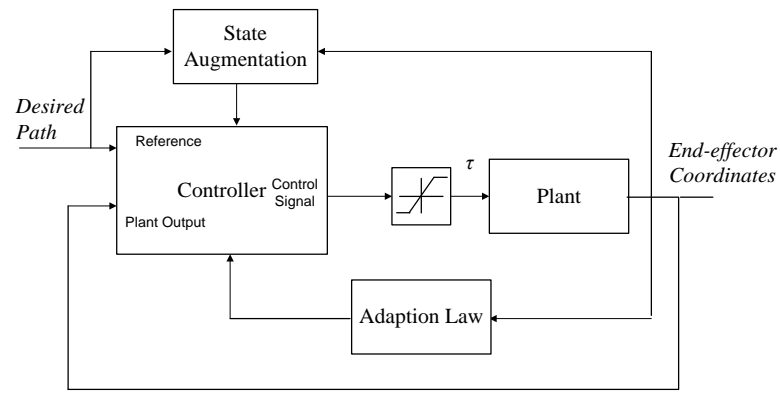

Fig. 2. The block diagram of the state augmented adaptive backstepping controller.

\section{State Augmented Adaptive Backstepping Control}

Some of the dynamical system parameter values are rarely known or may be variable. In adaptive backstepping approach the parameters vector $\theta$ is assumed to be unknown and is replaced by $\hat{\theta}$ which is the estimation of $\theta$. In order to expand the idea of state augmented backstepping controller to the adaptive case, the control law (23) is modified by replacing $\theta$ by $\hat{\theta}$ and the adaptive control signal is,

$$
\begin{aligned}
\tau=-\hat{\theta} \varphi-M k p x_{3}-M k_{i} x_{2}-x_{2} \\
-k_{d}\left(x_{3}+k_{p} x_{2}+k_{i} x_{1}\right)
\end{aligned}
$$

Fig. 2 presents the block diagram of the state augmented adaptive control approach. The new Lyapunov function is defined as,

$$
V_{5}\left(x_{1}, x_{2}, x_{3}, \hat{\theta}\right)=V_{4}\left(x_{1}, x_{2}, x_{3}\right)+\frac{1}{2 \gamma} \tilde{\theta}^{2}
$$

Which is a positive-definite function by considering $\gamma$ as a positive gain. $\tilde{\theta}$ is defined as $\tilde{\theta}=\theta-\hat{\theta}$. By taking the derivative of the Lyapunov function,

$$
\dot{V}_{5}=\dot{V}_{4}+\frac{1}{\gamma} \tilde{\theta} \dot{\tilde{\theta}}
$$

Substituting (25) in (27) will result in,

$$
\begin{aligned}
\dot{V}_{5}=\tilde{\theta} \varphi\left(x_{3}\right. & \left.+k_{p} x_{2}+k_{i} x_{1}\right)-k_{p} x_{2}{ }^{2} \\
& -k_{d}\left(x_{3}+k_{p} x_{2}+k_{i} x_{1}\right)^{2}+\frac{1}{\gamma} \tilde{\theta} \dot{\tilde{\theta}}
\end{aligned}
$$


The parameter adaption law is defined as,

$$
\dot{\hat{\theta}}=\gamma \varphi\left(x_{3}+k_{p} x_{2}+k_{i} x_{1}\right)
$$

By substituting (29) in (28),

$$
\dot{V}_{5}=-k_{p} x_{2}{ }^{2}-k_{d}\left(x_{3}+k_{p} x_{2}+k_{i} x_{1}\right)^{2}
$$

Which is a negative function. So based on Theorem I. and according to (26) and (30), defining the control signal as (25) and the parameters adaption law as (29) will stabilize the system.

\section{RESUlTS AND DISCUSSION}

The 2-DOF robot manipulator presented in Fig. 1 is regarded as the case study. The dynamic parameter values of the system are selected based on the information provided in [16]. These parameter values are not exactly known. The proposed controller should confront this problem and satisfy the system stability as well as zero tracking error. The backstepping, state augmented backstepping and state augmented adaptive backstepping controllers are applied to the system and the path tracking results are compared.

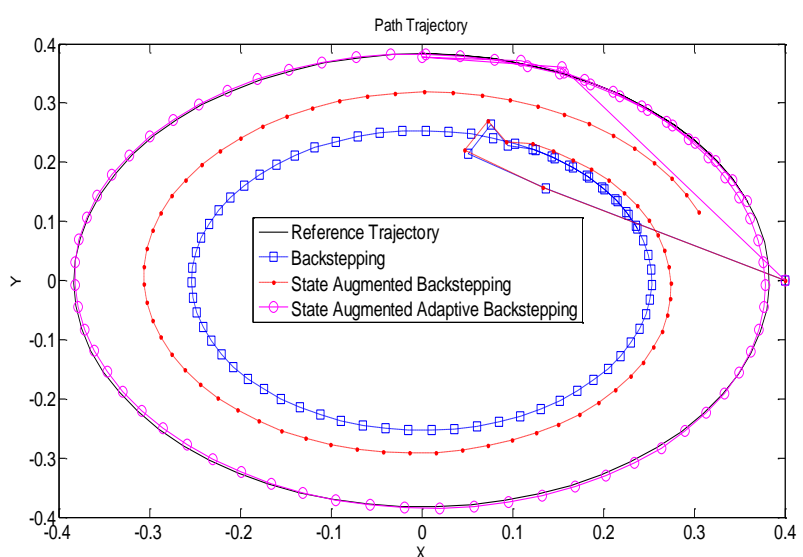

Fig. 3. The path tracking of the robot end-effector in $\mathrm{X}-\mathrm{Y}$ plane by applying different controllers.

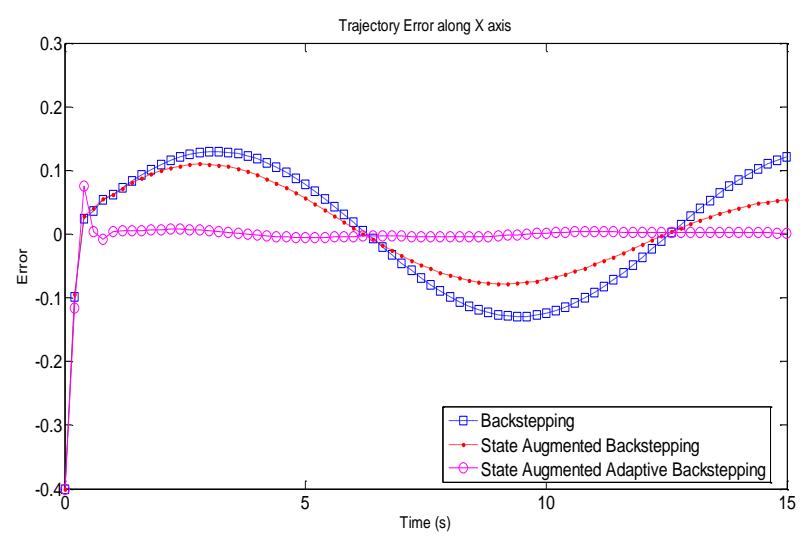

Fig. 4. The end-effector path trajectory error along $\mathrm{X}$ axis.

Fig. 3 presents the path trajectory of the robot end-effector by applying the control approaches. It is obvious that the state augmented adaptive backstepping has the best performance comparing to the other controllers.

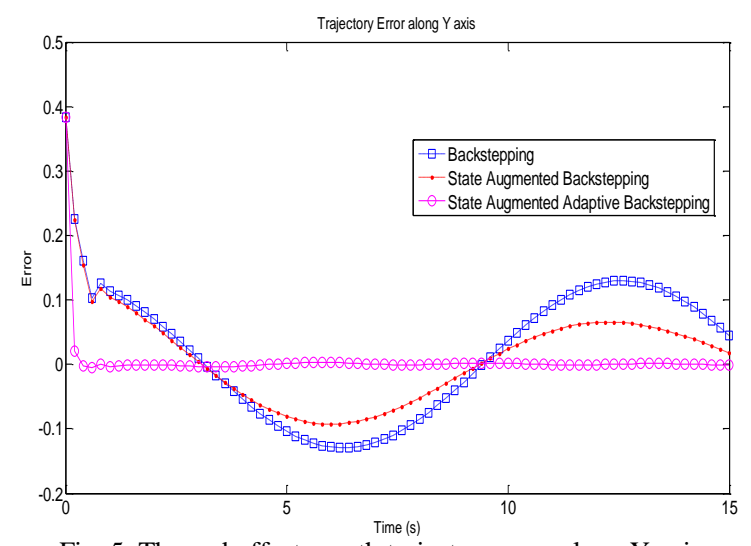

Fig. 5. The end-effector path trajectory error along Y axis.

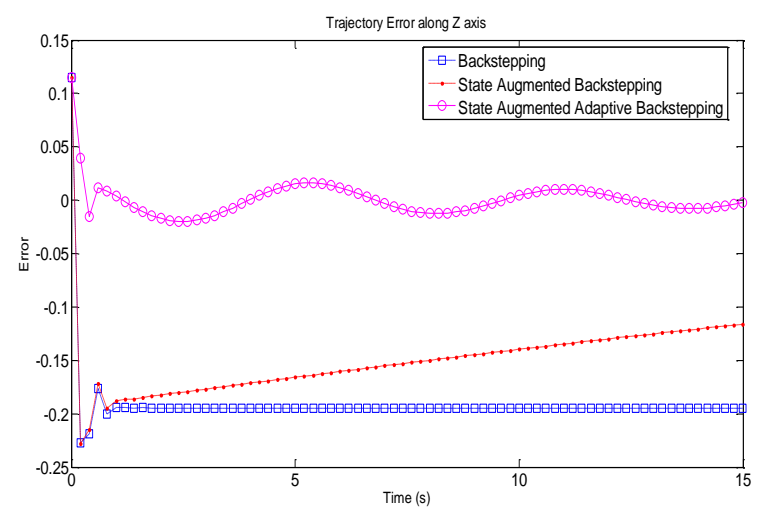

Fig. 6. The end-effector path trajectory error along $\mathrm{Z}$ axis.

In order to make a better comparison between controller performances, the trajectory error is analyzed along $\mathrm{X}, \mathrm{Y}$ and $\mathrm{Z}$ axis. The error signals are presented in Figs. 4, 5 and 6 respectively.

As the figures depict the state augmented adaptive backstepping shows better performance in controlling the system. Despite parameter uncertainties, zero tracking error is satisfied by applying the controller. Backstepping and state augmented backstepping control approaches are not able to make the end-effector track the desired path precisely and in front of system uncertainties they exhibit variations around the origin. In the case of state augmented adaptive backstepping controller the variations appear just along the $\mathrm{z}$-axis which decrease by decreasing the controller $k_{i}$ gain.

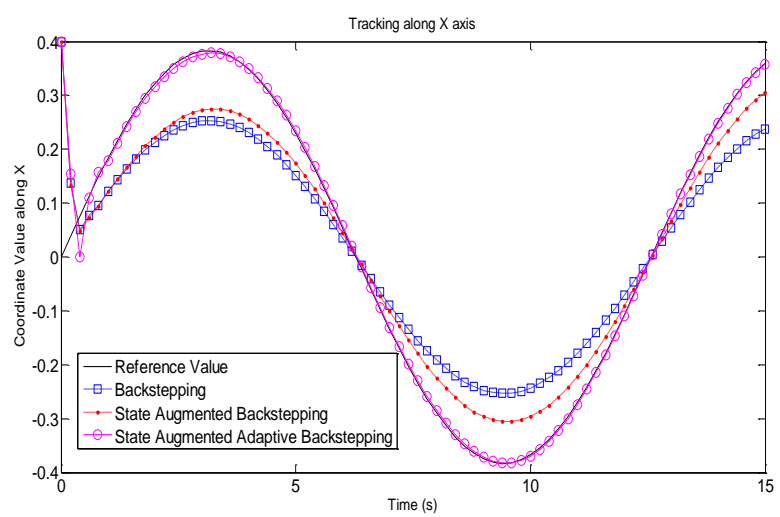

Fig. 7. End-effector position along $X$ axis.

The reference coordinate values as well as end-effector position along each axis are illustrated in Figs. 7, 8 and 9 respectively. The results prove the superiority of state augmented adaptive backstepping in controlling the system in 
presence of parameter uncertainties.

Another important issue is the control signal. Fig. 10 presents the control signal for both joints by applying the state augmented adaptive backstepping control approach. According to the figures, despite system nonlinearity and parameter variation and uncertainties, the controlled system tracks the desired trajectory. These figures along with (26) and (30) and Fig. 3, prove the power of state augmented adaptive backstepping in stabilizing and controlling the manipulator.

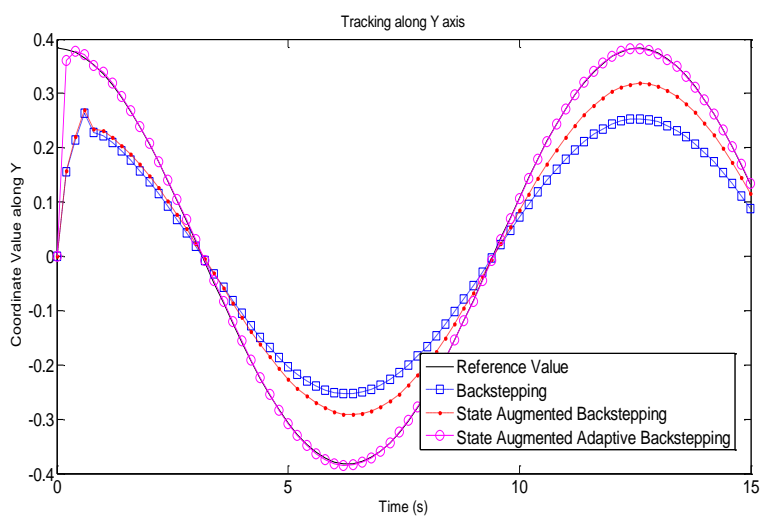

Fig. 8. End-effector position along Y axis.

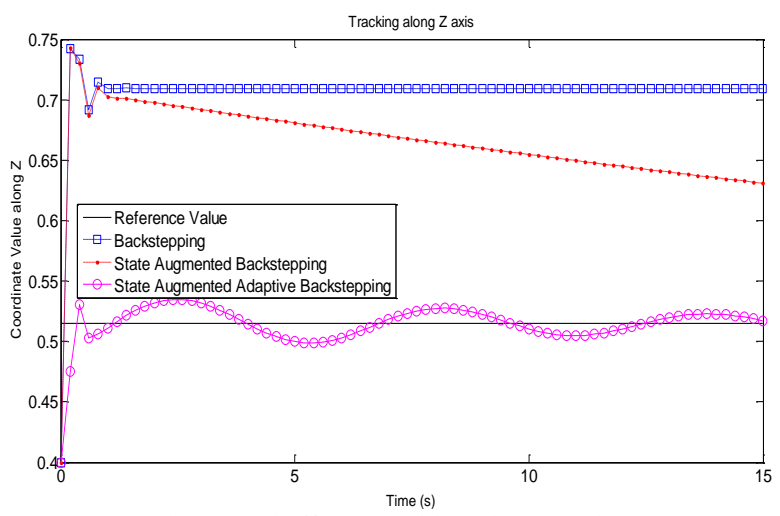

Fig. 9. End-effector position along $\mathrm{Z}$ axis.

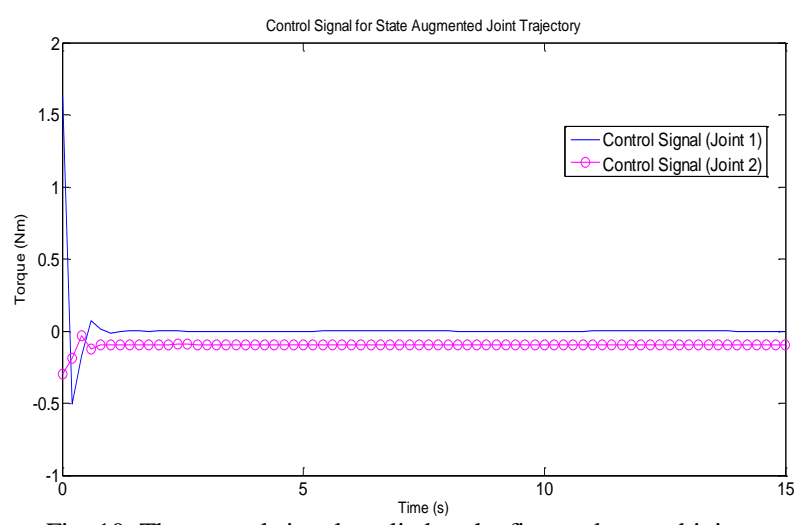

Fig. 10. The control signal applied to the first and second joints.

\section{CONCLUSION}

A successful attempt has been made in order to develop the state augmented adaptive backstepping for a 2-DOF robot manipulator. Dynamic model for the manipulator is presented and simulated. The error equation is derived and an additional state is augmented to the equations in order to improve the performance. The backstepping controller is designed recursively and a Lyapunov function is introduced which satisfies the stability of the augmented system. The controller structure is then extended to the state augmented adaptive backstepping controller. The adaption law and control signal are derived in a way to guarantee the system stability as well as good tracking performance. A comparison between the control approaches is made considering the end-effector path tracking problem. Based on the comparison, state augmented adaptive backstepping shows the best performance regarding the tracking error along each axis. Despite the system nonlinearity and parameter uncertainties the state augmented adaptive backstepping was able to control the system and make the end-effector track the desired path. A variety of reference signals are considered along each axis which demonstrate the efficiency of state augmented adaptive backstepping controller in making the manipulator track both sinusoidal and step signals. Stabilizing the system along with the good tracking performance make the state augmented adaptive backstepping a good candidate for many complicated dynamical systems.

\section{ACKNOWLEDGMENT}

The authors thank Dr. Vahid Azimirad and his M.Sc. student, Ms. Leila Mehri for the design and construction of the 2-DOF robot manipulator and providing the robot information.

\section{REFERENCES}

[1] S. Ueki, H. Kawasaki et al., "Development of a hand-assist robot with multi-degrees-of-freedom for rehabilitation therapy," IEEE/ASME Trans. Mechatronics, vol. 17, no. 1, pp. 136-146, 2012

[2] J. Huang, X. Zhang et al., "A High-integrated and high-precision robot manipulator joint servo system," in Proc. 11th World Congress on Intelligent Control and Automation, WCICA, IEEE, Sheyang, 2014 pp. 48-52.

[3] T. Haidegger, M. Barreto et al., "Applied ontologies and standards for service robots," Robotics and Autonomous Systems, vol. 61, no. 11, pp 1215-1223, 2013.

[4] G. Bekey, Current Trends in Robotics: Technology and Ethics. Robot Ethics: The Ethical and Social Implications of Robotics, Cambridge: MIT Press, 2012, pp. 17-34.

[5] J. Maneeratanaporn, P. Patompak, S. Varongkriengkrai, I. Nilkhamhang, and K. Tungpimolrut, "Adaptive backstepping controller for triple rotary joint manipulator," in Proc. SICE Annual Conference, Taipei, 2010, pp. 431-435.

[6] K. Lochan and B. K. Roy, "Control of two-link 2-dof robot manipulator using fuzzy logic techniques: a review," in Proc. Fourth International Conference on Soft Computing for Problem Solving, Springer, India, pp. 499-511, 2015.

[7] N. Nikdel, P. Nikdel, M. A. Badamchizadeh, and I. Hassanzadeh, "Using neural network model predictive control for controlling shape memory alloy-based manipulator," IEEE Trans. Industrial Electronics, vol. 61, no. 3, pp. 1394-1401, 2014.

[8] N. Nikdel, and M. A. Badamchizadeh, "Design and implementation of neural controllers for shape memory alloy-actuated manipulator," Journal of Intelligent Material Systems and Structures, vol. 26, no. 1, pp. 20-28, 2015.

[9] H. S. Shiromoto, V. Andrieu, and C. Prieur, "Combining a backstepping controller with a local stabilizer," in Proc. American Control Conference, San Francisco, CA, 2011, pp. 3197-3202.

[10] J. Liu, N. Zhan, and H. Zhao, "Automatically discovering relaxed Lyapunov functions for polynomial dynamical systems," Mathematics in Computer Science, vol. 6, no. 4, pp. 395-408, 2012.

[11] R. Hajihasani, V. Azimirad, Z. Boroujeni, and A. Jalali, "A new real-time adaptive control for teleoperation: simulation and experimental implementation," in Proc. Iran's Third International Conference on Industrial Automation, Iran, 2013. 
[12] W. Sun, H. Gao, and O. Kaynak, "Adaptive backstepping control for active suspension systems with hard constraints," IEEE/ASME Trans. Mechatronics, vol. 18, no. 3, pp. 1072-1079, 2013.

[13] Y. Zhu, H. Su, and M. Krstic, "Adaptive backstepping control of uncertain linear systems under unknown actuator delay," Automatica, vol. 54, pp. 256-265, 2015

[14] R. Skjetne and T. L. Fossen, "On integral control in backstepping: Analysis of different techniques," in Proc. the American Control Conference, Boston, USA, 2004, pp. 1899-1904.

[15] P. Corke. Denavit-Hartenberg Notation for Common Robots. [Online]. Available: http://petercorke.com/doc/rtb_dh.pdf

[16] M. A. Soltanmoradi, V. Azimirad, and M. Hajibabazadeh, "Detecting finger movement through classification of electromyography signals for use in control of robots," in Proc. Second RSI/ISM International Conference on Robotics and Mechatronics, 2014, pp. 791-794.

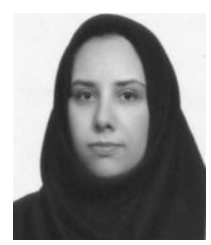

Nazila Nikdel Amand was born in Tehran, Iran, in May 1985. She received her B.S. and M.S. degrees in control engineering from University of Tabriz, Tabriz, Iran, in 2007 and 2010, respectively. She is currently pursuing the Ph.D. degree in Control Engineering, University of Tabriz.

Her research interests include robotics, brain-computer interface, teleoperation and adaptive control.

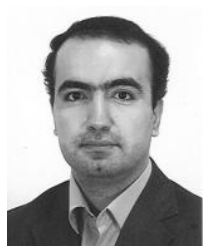

Mohammad Ali Badamchizadeh was born in Tabriz, Iran, in December 1975. He received his B.S. degree in electrical engineering and the M.Sc. and $\mathrm{PhD}$ degrees in control engineering from the University of Tabriz in 1998, 2001 and 2007 respectively.

$\mathrm{He}$ is currently an Associate Professor with the Faculty of Electrical and Computer Engineering, University of Tabriz. His research interests include adaptive control, hybrid dynamical systems, stability of systems, time-delay systems. 